\title{
Scaling and spatial intermittency of thermal dissipation in turbulent convection
}

Cite as: Phys. Fluids 31, 075104 (2019); https://doi.org/10.1063/1.5098073

Submitted: 31 March 2019 . Accepted: 17 June 2019 . Published Online: 16 July 2019

Shashwat Bhattacharya (D), Ravi Samtaney (D), and Mahendra K. Verma (i)

\section{ARTICLES YOU MAY BE INTERESTED IN}

Large eddy simulation of hydrodynamic turbulence using renormalized viscosity

Physics of Fluids 31, 065102 (2019); https://doi.org/10.1063/1.5096335

On heat transport and energy partition in thermal convection with mixed boundary conditions

Physics of Fluids 31, 066601 (2019); https://doi.org/10.1063/1.5095242

Taylor-Couette flow of shear-thinning fluids

Physics of Fluids 31, 053102 (2019); https://doi.org/10.1063/1.5088143

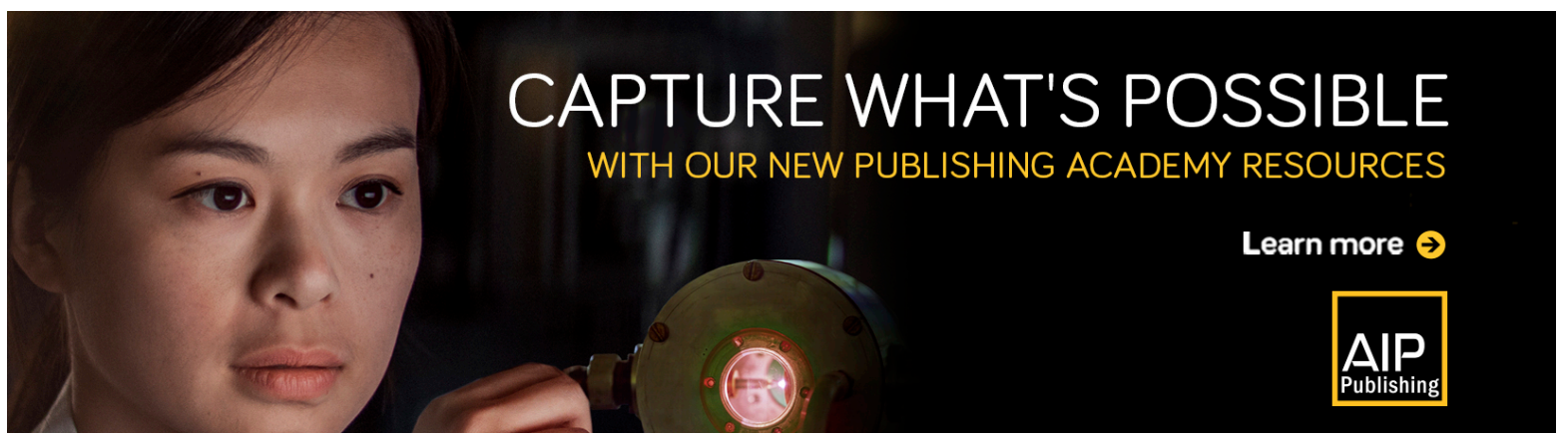




\title{
Scaling and spatial intermittency of thermal dissipation in turbulent convection
}

\author{
Cite as: Phys. Fluids 31, 075104 (2019); doi: 10.1063/1.5098073 \\ Submitted: 31 March 2019 - Accepted: 17 June 2019 • \\ Published Online: 16 July 2019
}

\author{
Shashwat Bhattacharya, ${ }^{1, a)}$ (D) Ravi Samtaney, ${ }^{2}$ (D) and Mahendra K. Verma ${ }^{3}$
}

\begin{abstract}
AFFILIATIONS
${ }^{1}$ Department of Mechanical Engineering, Indian Institute of Technology Kanpur, Kanpur 208016, India

${ }^{2}$ Mechanical Engineering, Division of Physical Science and Engineering, King Abdullah University of Science and Technology, Thuwal 23955, Saudi Arabia

${ }^{3}$ Department of Physics, Indian Institute of Technology Kanpur, Kanpur 208016, India
\end{abstract}

a) Electronic mail: shabhatt@iitk.ac.in

\begin{abstract}
We derive scaling relations for the thermal dissipation rate in the bulk and in the boundary layers for moderate and large Prandtl number (Pr) convection. Using direct numerical simulations of Rayleigh-Bénard convection, we show that the thermal dissipation in the bulk is suppressed compared to passive scalar dissipation. The suppression is stronger for large Pr. We further show that the dissipation in the boundary layers dominates that in the bulk for both moderate and large Pr. The probability distribution functions of thermal dissipation rate, both in the bulk and in the boundary layers, are stretched exponential, similar to passive scalar dissipation.
\end{abstract}

Published under license by AIP Publishing. https://doi.org/10.1063/1.5098073

\section{INTRODUCTION}

Scalar fields, such as temperature and concentration, are often carried along by turbulent flows. Flows with scalars are ubiquitous and frequently encountered in engineering and atmospheric applications. In general, these scalar fields influence the dynamics of fluid flow. The resulting coupling between the momentum and the scalar equations, along with strong nonlinearities, makes such flows very complex. Obukhov ${ }^{1}$ and Corrsin ${ }^{2}$ described the energetics of a simplified system consisting of homogeneous isotropic turbulence with passive scalar fields; such scalars do not affect the velocity field. In passive scalar turbulence, both kinetic energy [defined as $(1 / 2)\left\langle|\mathbf{u}|^{2}\right\rangle$ ] and scalar energy [defined as $(1 / 2)\left\langle\theta^{2}\right\rangle$ ] are supplied at large scales. Here, $\theta$ and $\mathbf{u}$ are scalar and velocity fields, respectively, and \langle\rangle denotes volume average. The supplied kinetic and scalar energies cascade to intermediate scales and then to dissipative scales. Similar to kinetic energy in homogeneous turbulence, the rate of scalar energy supply equals the scalar energy cascade rate $\Pi_{\theta}$ and the scalar dissipation rate $\epsilon_{\theta} \cdot{ }^{3,4}$ Dimensional analysis gives $\epsilon_{\theta} \approx U \Theta^{2} / L$, where $L, U$, and $\Theta$ are large-scale length, velocity, and scalar, respectively.

In the present work, we consider turbulence in buoyancydriven convection, which is an example of active scalar turbulence where the scalar field (temperature) influences the flow-dynamics. We focus on an idealized system called Rayleigh-Bénard convection (RBC) in which a fluid is enclosed between two horizontal walls, with the bottom wall being hotter than the top one..$^{5-7}$ Each horizontal wall is isothermal. RBC is specified by two nondimensional parameters-Rayleigh number (Ra) and Prandtl number (Pr). These parameters are defined as

$$
\mathrm{Ra}=\frac{\alpha g \Delta d^{3}}{v \kappa}, \quad \operatorname{Pr}=\frac{v}{\kappa},
$$

where $\alpha, v$, and $\kappa$, respectively, are the thermal expansion coefficient, kinematic viscosity, and thermal diffusivity of the fluid, $g$ is the gravitational acceleration, and $\Delta$ and $d$, respectively, are the temperature difference and the distance between the top and bottom plates.

The energetics of thermally driven convection is more complex than that of passive scalar turbulence; this is due to the two-way coupling between the governing equations of momentum and thermal energy (see Sec. II), along with the presence of thermal boundary layers. Presently, we focus on the properties of thermal dissipation rate $\epsilon_{T}(\mathbf{r})=\kappa(\nabla T)^{2}$, where $T$ is the temperature field. In RBC, the volume-averaged thermal dissipation rate is related to the Nusselt number $(\mathrm{Nu})$ by the following relation derived by Shraiman and 
Siggia:

$$
\epsilon_{T}=\left\langle\kappa(\nabla T)^{2}\right\rangle=\frac{\kappa \Delta^{2}}{d^{2}} \mathrm{Nu}=\frac{U \Delta^{2}}{d} \frac{\mathrm{Nu}}{\mathrm{Pe}} .
$$

The Nusselt number is the ratio of the total heat flux and the conductive heat flux, and $\mathrm{Pe}=U d / \kappa$ is the Péclet number. When the thermal boundary layers are less significant than the bulk (as in the ultimate regime proposed by Kraichnan ${ }^{9}$ ), or absent (as in a periodic box $^{10}$ ), both $\mathrm{Nu}$ and Pe are proportional to $\sqrt{\mathrm{RaPr}}$ (see Refs. 7, 11, and 12). These relations, when substituted in Eq. (1), yield $\epsilon_{T} \sim U \Delta^{2} / d$, similar to passive scalar turbulence.

In $\mathrm{RBC}$, the thermal boundary layers near the conducting walls play an important role in the scaling of thermal dissipation rate. In our present work, we focus on the Ra dependence of thermal dissipation rate and other quantities. For moderate Prandtl numbers (of order 1), it has been shown via scaling arguments, ${ }^{11,13-15}$ experiments, ${ }^{14,16-23}$ and numerical simulations ${ }^{24-31}$ that

$$
\mathrm{Pe} \sim \mathrm{Ra}^{0.5}, \quad \mathrm{Nu} \sim \mathrm{Ra}^{0.3} .
$$

Note that the exponents in the above expressions shown here are approximate. Substitution of these expressions into Eq. (1) yields

$$
\epsilon_{T} \sim \frac{\kappa \Delta^{2}}{d^{2}} \mathrm{Ra}^{0.3} \sim \frac{U \Delta^{2}}{d} \mathrm{Ra}^{-0.2} .
$$

When compared to passive scalar flow, the additional term $\mathrm{Ra}^{-0.2}$ in $\mathrm{RBC}$ accounts for suppression of nonlinear interactions due to the presence of walls; Pandey and Verma ${ }^{30}$ and Pandey et al. ${ }^{31}$ showed that in RBC, the ratio of the nonlinear term to the diffusive term in the equation for thermal energy is proportional to $\mathrm{PeRa}^{-0.30}$ instead of Pe. The walls truncate some of the Fourier modes, resulting in several channels of nonlinear interations and energy cascades to be blocked (see Ref. 4 for details). Consequently, thermal dissipation in RBC is weakened compared to free passive scalar turbulence. For large Pr, Pandey and Verma ${ }^{30}$ and Pandey et al. ${ }^{31}$ have shown that $\epsilon_{T} \sim\left(U \Delta^{2} / d\right) \mathrm{Ra}^{-0.25}$ instead of $U \Delta^{2} / d$.

To better understand the effects of walls, we need to study the behavior of thermal dissipation separately in the boundary layers and in the bulk. It is generally believed that dissipation (thermal or viscous) occurs predominantly in the boundary layers. ${ }^{32,33}$ However, phenomenological arguments and numerical results presented by Verma, Kumar, and Pandey imply that significant dissipation occurs also at large scales, i.e., in the bulk. Motivated by this, Bhattacharya et al. ${ }^{34}$ computed the viscous dissipation rate separately in the bulk and in the boundary layers for moderate Pr. Interestingly, they found the bulk dissipation to be greater, albeit marginally, than the boundary layer dissipation. On the other hand, the thermal dissipation for moderate Pr convection was shown to be dominant in the boundary layers; refer to the work of Verzicco and Camussi $^{24}$ and Zhang, Zhou, and Sun. ${ }^{3}$

In this paper, we conduct a more detailed analysis of thermal dissipation rate in the bulk and boundary layers not only for moderate Pr but also for large Pr convection. Note that the statistics of thermal dissipation for large $\mathrm{Pr}$ is less explored in the literature. We compare and quantify the total and average thermal dissipation rates in the bulk and in the boundary layers using scaling arguments and numerical simulations. We also examine the probability distribution functions (PDFs) of the thermal dissipation in these regions.
Our analysis is similar to that conducted by Bhattacharya et al. ${ }^{34}$ on viscous dissipation rate.

The outline of the paper is as follows. In Sec. II, we present the governing equations of RBC along with their nondimensionalization. We discuss the numerical method in Sec. III. In Sec. IV, we compute the thermal boundary layer thickness and present scaling arguments for the thermal dissipation rate in the bulk and in the boundary layers. We verify these scaling relations using our numerical results. We also study the spatial intermittency of thermal dissipation rate. Finally, we conclude in Sec. V.

\section{GOVERNING EQUATIONS}

In $\mathrm{RBC}$, under the Boussinesq approximation, the thermal diffusivity $(\kappa)$ and the kinematic viscosity $(v)$ are treated as constants. The density of the fluid is considered to be a constant except for the buoyancy term in the governing equations. Furthermore, the viscous dissipation term is considered to be small and is therefore dropped from the temperature equation. The governing equations of RBC are as follows: ${ }^{4,3}$

$$
\begin{gathered}
\frac{\partial \mathbf{u}}{\partial t}+(\mathbf{u} \cdot \nabla) \mathbf{u}=-\nabla p / \rho_{0}+\alpha g T \hat{z}+v \nabla^{2} \mathbf{u}, \\
\frac{\partial T}{\partial t}+(\mathbf{u} \cdot \nabla) T=\kappa \nabla^{2} T \\
\nabla \cdot \mathbf{u}=0
\end{gathered}
$$

where $\mathbf{u}$ and $p$ are the velocity and pressure fields, respectively, $T$ is the temperature field with respect to a reference temperature, $\alpha$ is the thermal expansion coefficient, $\rho_{0}$ is the mean density of the fluid, and $g$ is acceleration due to gravity.

Using $d$ as the length scale, $\sqrt{\alpha g \Delta d}$ as the velocity scale, and $\Delta$ as the temperature scale, we nondimensionalize Eqs. (3)-(5), which yields

$$
\begin{gathered}
\frac{\partial \mathbf{u}}{\partial t}+\mathbf{u} \cdot \nabla \mathbf{u}=-\nabla p+T \hat{z}+\sqrt{\frac{\mathrm{Ra}}{\operatorname{Pr}}} \nabla^{2} \mathbf{u}, \\
\frac{\partial T}{\partial t}+\mathbf{u} \cdot \nabla T=\frac{1}{\sqrt{\operatorname{RaPr}}} \nabla^{2} T, \\
\nabla \cdot \mathbf{u}=0 .
\end{gathered}
$$

In Sec. III, we describe the numerical method used for our simulations.

\section{NUMERICAL METHOD}

We conduct our numerical analysis for (i) $\mathrm{Pr}=1$ and (ii) $\mathrm{Pr}$ $=100$ fluids. For $\operatorname{Pr}=1$, we use the simulation data of Bhattacharya et al. $^{34}$ and Kumar and Verma, ${ }^{37}$ which were obtained using the finite volume code OpenFOAM. ${ }^{38}$ The simulations were conducted on a $256^{3}$ grid for Ra ranging from $10^{6}$ to $10^{8}$. No-slip boundary conditions were imposed at all the walls, isothermal boundary conditions at the top and bottom walls, and adiabatic boundary conditions at the sidewalls. For time marching, second-order Crank-Nicholson scheme was used. For $\operatorname{Pr}=100$, we conduct fresh simulations following the aforementioned schemes, boundary conditions, and grid 
TABLE I. Details of our numerical data obtained using direct numerical simulations performed in a cubical box: the Prandtl number $(\mathrm{Pr})$, the Rayleigh number $(\mathrm{Ra})$, the Péclet number $(\mathrm{Pe})$, the time step $(\Delta t)$, the ratio of the Batchelor length scale ${ }^{41}$ $\left(\eta_{\theta}\right)$ to the maximum mesh width $\Delta x_{\max }$, the Nusselt number $(\mathrm{Nu})$, the Nusselt number $\left(\mathrm{Nu} \mathrm{u}_{\mathrm{S}}\right)$ deduced from $\epsilon_{T}$ using Eq. (1), the ratio of the thermal boundary layer thickness $\delta_{T}$ to the cell height $d$, the number of grid points in the thermal boundary layer $\left(N_{\mathrm{BL}}\right)$, and the number of snapshots over which the quantities are averaged.

\begin{tabular}{lccccccccc}
\hline \hline $\operatorname{Pr}$ & $\mathrm{Ra}$ & $\mathrm{Pe}$ & $\Delta t$ & $\eta_{\theta} / \Delta x_{\max }$ & $\mathrm{Nu}$ & $\mathrm{Nu}_{S}$ & $\delta_{T} / d$ & $N_{\mathrm{BL}}$ & Snapshots \\
\hline 1 & $1 \times 10^{6}$ & 150 & $1 \times 10^{-3}$ & 3.6 & 8.40 & 8.26 & 0.061 & 23 & 56 \\
1 & $2 \times 10^{6}$ & 212 & $1 \times 10^{-3}$ & 2.8 & 10.1 & 10.1 & 0.050 & 19 & 56 \\
1 & $5 \times 10^{6}$ & 342 & $1 \times 10^{-3}$ & 2.1 & 13.3 & 13.4 & 0.037 & 14 & 55 \\
1 & $1 \times 10^{7}$ & 460 & $1 \times 10^{-3}$ & 1.7 & 16.0 & 16.1 & 0.031 & 12 & 100 \\
1 & $2 \times 10^{7}$ & 654 & $1 \times 10^{-3}$ & 1.3 & 20.0 & 19.7 & 0.025 & 10 & 100 \\
1 & $5 \times 10^{7}$ & 1080 & $1 \times 10^{-3}$ & 1.0 & 25.5 & 25.7 & 0.019 & 8 & 101 \\
1 & $1 \times 10^{8}$ & 1540 & $1 \times 10^{-3}$ & 0.8 & 32.8 & 32.0 & 0.016 & 7 & 86 \\
100 & $2 \times 10^{6}$ & 277 & $5 \times 10^{-4}$ & 2.8 & 11.1 & 11.1 & 0.045 & 17 & 41 \\
100 & $5 \times 10^{6}$ & 496 & $1 \times 10^{-3}$ & 2.0 & 14.5 & 14.4 & 0.034 & 13 & 50 \\
100 & $1 \times 10^{7}$ & 698 & $1 \times 10^{-3}$ & 1.6 & 17.2 & 17.1 & 0.029 & 12 & 52 \\
100 & $2 \times 10^{7}$ & 1036 & $1 \times 10^{-3}$ & 1.3 & 20.1 & 20.3 & 0.025 & 10 & 99 \\
100 & $5 \times 10^{7}$ & 1772 & $1 \times 10^{-3}$ & 1.0 & 26.0 & 26.0 & 0.019 & 8 & 101 \\
\hline \hline
\end{tabular}

resolution for Ra's ranging from $2 \times 10^{6}$ to $5 \times 10^{7}$. A constant time step was chosen, with $\Delta t=10^{-3}$ and $5 \times 10^{-4}$, depending on the parameters (see Table I for details). Here, $t=1$ corresponds to $d / \sqrt{\alpha g \Delta d}$.

We ensure that a minimum of 8 grid points is in the thermal boundary layers, thereby satisfying the resolution criterion set by Grötzbach ${ }^{39}$ and Verzicco and Camussi. ${ }^{24}$ In RBC, the thermal boundary layer thickness $\delta_{T}$ is defined as the distance between the wall and the point where the tangent to the planar-averaged temperature profile near the wall intersects with the average bulk temperature line. ${ }^{5,25,26,40}$ To ensure that the smallest length scales are resolved, we note that the ratio of the Batchelor length scale ${ }^{41} \eta_{\theta}$ $=\left(v \kappa^{2} / \epsilon_{u}\right)^{1 / 4}$ to the maximum mesh width $\Delta x_{\max }$ remains greater than unity for all runs. The only exception is for the $\mathrm{Ra}=10^{8}, \mathrm{Pr}$ $=1$ case where $\eta_{\theta}=0.8$, which is marginally less than unity. The Nusselt numbers computed using our data are consistent with those obtained in other simulations of RBC for the same geometry; ${ }^{30,31,42}$ this is how we validate our data. Furthermore, the Nusselt numbers computed numerically using $\left\langle u_{z} T\right\rangle$ match closely with those computed using $\epsilon_{T}$ and Eq. (1). This further validates our simulations. See Table I for the comparison of these two Nusselt numbers. All the quantities analyzed in this work are time-averaged over 40-100 snapshots after attaining a steady-state (see Table I).

In Sec. IV, we discuss the numerical results, focusing on the scaling of the thermal dissipation rate in the bulk and in the boundary layers, their relative contributions to the total thermal dissipation rate, and their spatial intermittency.

\section{NUMERICAL RESULTS}

\section{A. Boundary layer thickness}

Using the simulation results, we first compute the thickness of the thermal boundary layers. Theoretically, boundary layer thickness $\left(\delta_{T}\right)$ is related to the Nusselt number as

$$
\frac{\delta_{T}}{d}=\frac{1}{2 \mathrm{Nu}} \text {. }
$$

Now, as discussed in Sec. I, Nu $\sim \mathrm{Ra}^{0.3}$ for Pr of order 1. Numerical simulations $s^{30,31,43}$ reveal that $\mathrm{Nu} \sim \mathrm{Ra}^{0.3}$ for large $\mathrm{Pr}$ as well. Therefore, for both $\operatorname{Pr}=1$ and 100, we expect

$$
\frac{\delta_{T}}{d} \sim \mathrm{Ra}^{-0.3}
$$

We numerically compute $\delta_{T}$ 's using the planar averaged temperature profile and list them in Table I. Furthermore, we plot them vs Ra in Figs. 1(a) and 1(b) for both $\operatorname{Pr}=1$ and 100. The best-fit curves of the data yield

$$
\frac{\delta_{T}}{d}= \begin{cases}3.6 \mathrm{Ra}^{-0.30}, & \operatorname{Pr}=1, \\ 2.4 \mathrm{Ra}^{-0.28}, & \operatorname{Pr}=100,\end{cases}
$$

with the error in the exponents being approximately 0.01 . The obtained fit is reasonably consistent with Eq. (10).

\section{B. Scaling of thermal dissipation rate}

In this subsection, we study the scaling of average thermal dissipation rate in the bulk $\left(\epsilon_{T, \text { bulk }}\right)$ and in the boundary layers $\left(\epsilon_{T, \mathrm{BL}}\right)$ using our numerical data. These quantities are dissipation per unit volume. Based on these, using scaling arguments, we predict the relations for the total dissipation rate in the bulk $\left(\tilde{D}_{T, \text { bulk }}\right)$ and in the boundary layers $\left(\tilde{D}_{T, \mathrm{BL}}\right)$, which are the products of average thermal dissipation rates in these regions and their corresponding volumes. We verify their scaling relations using our simulation data and analyze the relative strength of the bulk and the boundary layer dissipation.

\section{Bulk dissipation}

Using our simulation data, we numerically compute $\epsilon_{T, \text { bulk }}$ $=\left\langle\kappa|\nabla T(\mathbf{r})|^{2}\right\rangle_{\text {bulk }}$ and the large-scale mean flow $U=\sqrt{\left\langle|\mathbf{u}(\mathbf{r})|^{2}\right\rangle}$. 

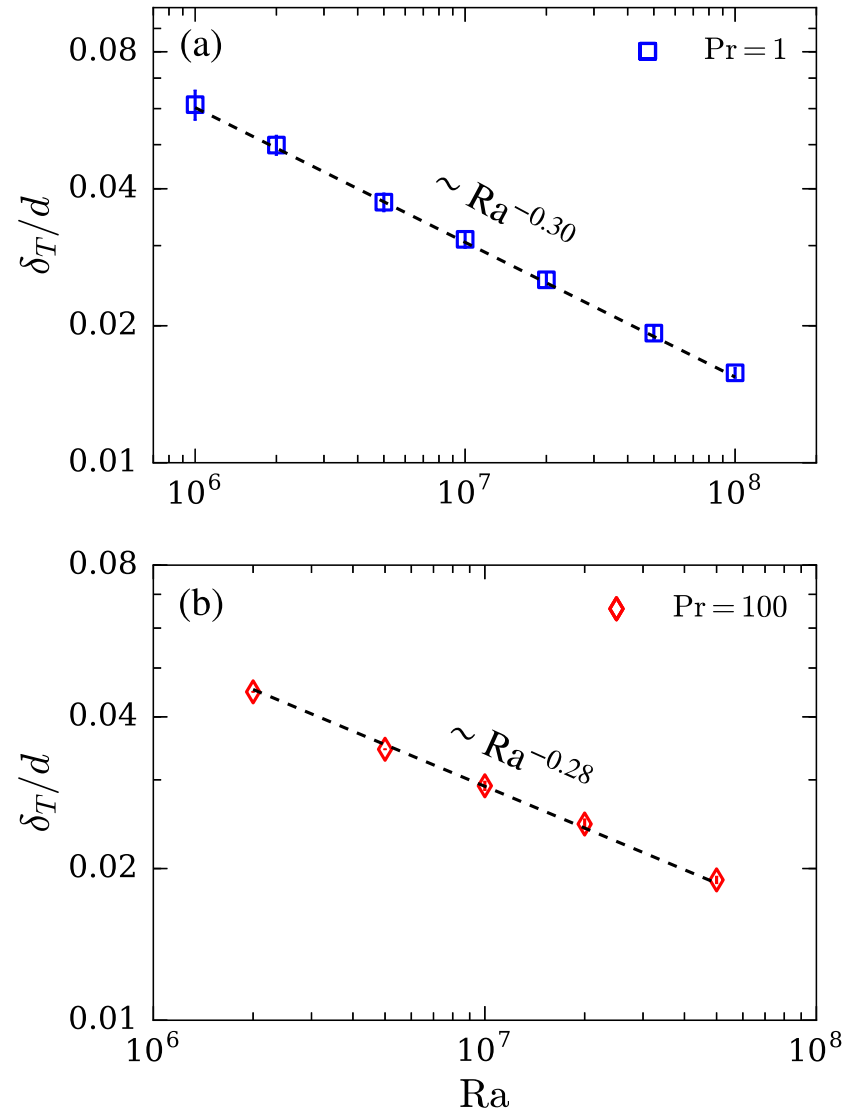

FIG. 1. For (a) $\operatorname{Pr}=1$ and (b) $\operatorname{Pr}=100$ : plots of normalized thermal boundary layer thickness $\delta_{T} / d$ vs $\mathrm{Ra}$, along with $\mathrm{Ra}^{-0.30}$ and $\mathrm{Ra}^{-0.28}$ fits (dashed curves). The error-bars represent the standard deviation of the dataset with respect to the temporal average.

In deriving their unifying scaling theory, Grossmann and Lohse $\mathrm{e}^{11,12}$ argued that $\epsilon_{T, \text { bulk }} \sim U \Delta^{2} / d$. However, from our numerical data, we observe that

$$
\epsilon_{T, \text { bulk }} \sim \begin{cases}\left(U \Delta^{2} / d\right) \mathrm{Ra}^{-0.22}, & \operatorname{Pr}=1, \\ \left(U \Delta^{2} / d\right) \mathrm{Ra}^{-0.25}, & \operatorname{Pr}=100,\end{cases}
$$

instead of $U \Delta^{2} / d$ (see Fig. 2). The errors in the exponents are 0.02 and 0.01 for $\operatorname{Pr}=1$ and 100 , respectively. Thus, the thermal dissipation in the bulk in RBC scales similar to the dissipation in the entire volume and is distinctly weaker than that in passive scalar turbulence. For moderate Pr fluids, the decrease in $\epsilon_{T, \text { bulk }} /\left(U \Delta^{2} / d\right)$ with $\mathrm{Ra}$ has also been observed by Emran and Schumacher ${ }^{44}$ and Verzicco and Camussi ${ }^{24}$ for convection in a cylindrical cell and by Zhang, Zhou, and Sun $^{35}$ for two-dimensional RBC. As discussed in Sec. I, the walls suppress nonlinear interactions in RBC, ${ }^{30,31}$ consequently weakening the thermal dissipation rate at large scales. Note that Bhattacharya et al. ${ }^{34}$ observed similar suppression of viscous dissipation in the bulk, where $\epsilon_{u \text {, bulk }} \sim\left(U^{3} / d\right) \mathrm{Ra}^{-0.18}$ instead of $U^{3} / d$ for $\operatorname{Pr}=1$.

The aforementioned suppression has an important implication in the scaling of the total thermal dissipation in the bulk $\left(\tilde{D}_{T, \text { bulk }}\right)$.

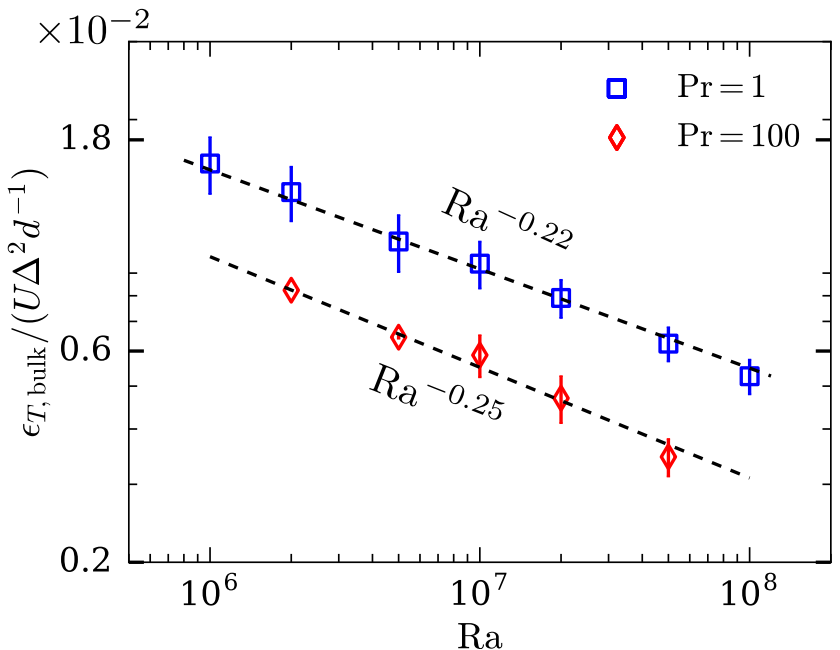

FIG. 2. Plots of average thermal dissipation rate in the bulk, normalized with $U \Delta^{2} / d$, vs $\mathrm{Ra}$. The bulk dissipation is distinctly weaker than $U \Delta^{2} / d$. The error bars represent the standard deviation of the dataset with respect to the temporal average.

The bulk volume can be approximated as

$$
V_{\text {bulk }}=\left(d-2 \delta_{T}\right) d^{2} \approx d^{3},
$$

because $\delta_{T} \ll d$ (see Table I). We will now derive the scaling relations for $\tilde{D}_{T, \text { bulk }}$ separately for $\operatorname{Pr}=1$ and 100 .

1. $\operatorname{Pr}=1$ : Using Eqs. (12) and (13), we write the following for the bulk dissipation:

$$
\tilde{D}_{T, \text { bulk }}=\epsilon_{T, \text { bulk }} V_{\text {bulk }} \sim\left(\frac{U \Delta^{2}}{d} \mathrm{Ra}^{-0.22}\right) d^{3} .
$$

By multiplying the numerator and the denominator of the rightmost expression in Eq. (14) by $d / \kappa$, we rewrite $\tilde{D}_{T \text {, bulk }}$ as

$$
\left(\frac{U \Delta^{2}}{d} \mathrm{Ra}^{-0.22}\right) d^{3}=\left(\kappa \Delta^{2} d\right) \mathrm{PeRa}^{-0.22},
$$

where $\mathrm{Pe}=U d / \kappa$ is the Péclet number. As discussed in Sec. I, Pe $\sim \mathrm{Ra}^{0.5}$ for moderate Pr. Substituting this relation in Eqs. (14) and (15), we obtain

$$
\tilde{D}_{T, \text { bulk }} \sim\left(\kappa \Delta^{2} d\right) \mathrm{Ra}^{0.28} .
$$

2. $\operatorname{Pr}=100$ : Applying a similar procedure, we can write the total dissipation in the bulk for $\operatorname{Pr}=100$ as

$$
\tilde{D}_{T, \text { bulk }} \sim\left(\kappa \Delta^{2} d\right) \mathrm{PeRa}^{-0.25},
$$

because $\epsilon_{T, \text { bulk }} \sim\left(U \Delta^{2} / d\right) \mathrm{Ra}^{-0.25}$ in this case. Now, according to the predictions of Grossmann and Lohse ${ }^{12}$ and Shishkina et al. ${ }^{45}$ for large $\mathrm{Pr}$ convection, $\mathrm{Pe} \sim \mathrm{Ra}^{3 / 5}$. Pandey, Verma, and Mishra, ${ }^{43}$ Pandey and Verma, ${ }^{30}$ and Pandey et al. ${ }^{31}$ have also shown that for large $\mathrm{Pr}, \mathrm{Pe} \sim \mathrm{Ra}^{0.6}$. Substituting this relation in 
Eq. (17), we obtain

$$
\tilde{D}_{T, \text { bulk }} \sim\left(\kappa \Delta^{2} d\right) \mathrm{Ra}^{0.35} .
$$

Thus, the suppression of thermal dissipation in the bulk leads to a weaker dependence of the total thermal dissipation with Ra. Note that in the absence of this suppression, $\tilde{D}_{T, \text { bulk }} \sim\left(\kappa \Delta^{2} d\right)$ Pe. Had this been the case, $\tilde{D}_{T, \text { bulk }}$, normalized with $\kappa \Delta^{2} d$, would have been proportional to $\mathrm{Ra}^{0.5}$ for $\mathrm{Pr}=1$ and $\mathrm{Ra}^{0.6}$ for $\mathrm{Pr}=100$.

\section{Boundary layer dissipation}

The heat transport in the boundary layers is primarily diffusive due to steep temperature gradients. Thus, we expect the thermal dissipation in the boundary layers to be given by

$$
\epsilon_{T, \mathrm{BL}} \sim \kappa \Delta^{2} / \delta_{T}^{2} .
$$

We verify this by plotting the numerically computed $\epsilon_{T, \mathrm{BL}} /\left(\kappa \Delta^{2} / \delta_{T}^{2}\right)$ vs $\mathrm{Ra}$ in Fig. 3, where we observe the slope to be flat. For $\mathrm{Pr}$ $=100$ and at lower Ra, however, there is a very slight decrease in $\epsilon_{T, \mathrm{BL}} /\left(\kappa \Delta^{2} / \delta_{T}^{2}\right)$ with Ra. However, we will ignore this in our scaling analysis.

The total thermal dissipation in the boundary layers is given by $\tilde{D}_{T, \mathrm{BL}}=\epsilon_{T, \mathrm{BL}} V_{\mathrm{BL}}$. Substituting Eq. (19) in the above relation and noting that $V_{\mathrm{BL}}=2 \delta_{T} d^{2}$, we obtain

$$
\tilde{D}_{T, \mathrm{BL}} \sim\left(\frac{\kappa \Delta^{2}}{\delta_{T}^{2}}\right) \delta_{T} d^{2} \sim \kappa \Delta^{2} d\left(\frac{d}{\delta_{T}}\right) .
$$

As discussed in Sec. IV A, $\delta_{T} / d \sim \mathrm{Ra}^{-0.30}$ for $\mathrm{Pr}=1$ and $\sim \mathrm{Ra}^{-0.28}$ for $\operatorname{Pr}=100$. Substituting these relations in Eq. (20), we obtain

$$
\tilde{D}_{T, \mathrm{BL}} \sim \begin{cases}\left(\kappa \Delta^{2} d\right) \mathrm{Ra}^{0.30}, & \operatorname{Pr}=1, \\ \left(\kappa \Delta^{2} d\right) \mathrm{Ra}^{0.28}, & \operatorname{Pr}=100 .\end{cases}
$$

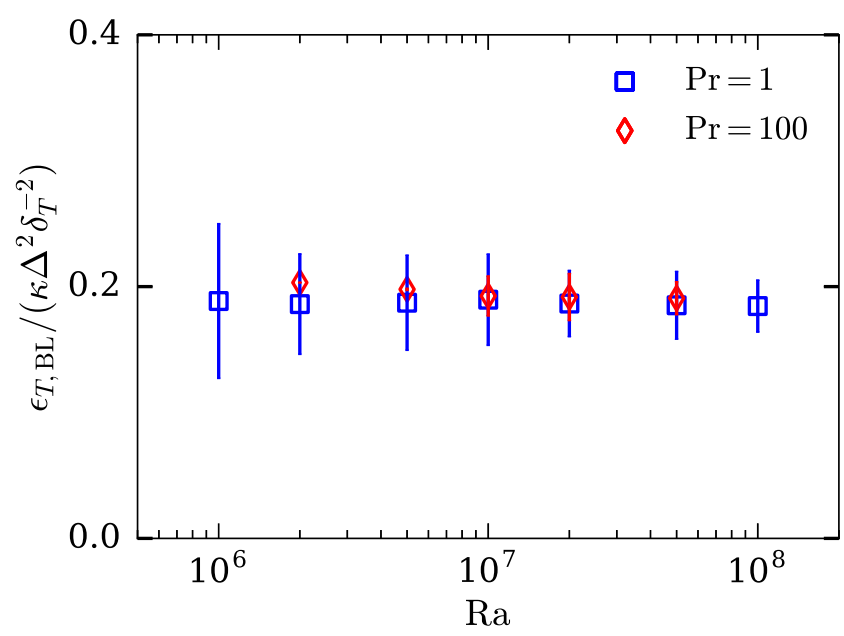

FIG. 3. Plots of average thermal dissipation in the boundary layers, normalized with $\kappa \Delta^{2} / \delta_{T}^{2}$, vs Ra. The error bars represent the standard deviation of the dataset with respect to the temporal average.

\section{Ratio of the boundary layer and the bulk dissipation}

To analyze the relative strengths of the thermal dissipation in the bulk and in the boundary layers, we divide Eq. (21) with Eqs. (16) and (18) to obtain the ratio of the total dissipation in the boundary layers and the bulk for $\operatorname{Pr}=1$ and 100, respectively. The predicted ratio is

$$
\frac{\tilde{D}_{T, \mathrm{BL}}}{\tilde{D}_{T, \text { bulk }}} \sim \begin{cases}\mathrm{Ra}^{0.02}, & \mathrm{Pr}=1, \\ \mathrm{Ra}^{-0.07}, & \mathrm{Pr}=100 .\end{cases}
$$

Thus, we expect the ratio of the boundary layer and bulk dissipation to have a weak dependence on $\mathrm{Ra}$. For $\mathrm{Pr}=1$, this ratio remains approximately constant, implying that the relative strengths of the bulk and the boundary layer dissipation remain roughly invariant with $\mathrm{Ra}$. However, for $\mathrm{Pr}=100$, the above ratio decreases weakly with $\mathrm{Ra}$; this implies that the relative strength of the boundary layer dissipation decreases with $\mathrm{Ra}$ and that of the bulk dissipation increases with $\mathrm{Ra}$. The magnitudes of the prefactors in Eq. (22) determine whether the bulk or the boundary layer dissipation is dominant. These prefactors are obtained using numerical simulations.

\section{Numerical verification of the scaling arguments}

We numerically verify the scaling relations predicted by Eqs. (16), (18), (21), and (22). We compute $\tilde{D}_{T}$ (the total dissipation in the entire volume), $\tilde{D}_{T, \text { bulk }}$, and $\tilde{D}_{T, \mathrm{BL}}$ using our simulation data and plot them vs Ra in Fig. 4(a) for Pr $=1$ and in Fig. 4(b) for $\operatorname{Pr}=100$. Our data fit well with the following expressions:

$$
\begin{gathered}
\tilde{D}_{T}=0.16 c \mathrm{Ra}^{0.29}, \quad \operatorname{Pr}=1,100, \\
\tilde{D}_{T, \text { bulk }}= \begin{cases}0.041 c \mathrm{Ra}^{0.29}, & \operatorname{Pr}=1, \\
0.015 c \mathrm{Ra}^{0.34}, & \operatorname{Pr}=100,\end{cases} \\
\tilde{D}_{T, \mathrm{BL}}= \begin{cases}0.12 c \mathrm{Ra}^{0.29}, & \operatorname{Pr}=1, \\
0.15 c \mathrm{Ra}^{0.28}, & \mathrm{Pr}=100,\end{cases}
\end{gathered}
$$

where $c=\kappa \Delta^{2} d$. The errors in the exponents in the above expressions range from 0.001 to 0.02 . The above expressions match with the scaling arguments presented in Eqs. (21), (16), and (18) within the fitting error. tion is

The computed ratio of the boundary layer and the bulk dissipa-

$$
\frac{\tilde{D}_{T, \mathrm{BL}}}{\tilde{D}_{T, \text { bulk }}} \approx \begin{cases}3.0, & \mathrm{Pr}=1, \\ 10 \mathrm{Ra}^{-0.06}, & \mathrm{Pr}=100,\end{cases}
$$

which agrees well with Eq. (22). We plot this ratio in Fig. 4(c) for $\mathrm{Pr}$ $=1$ and Fig. 4(d) for $\operatorname{Pr}=100$.

Because of the prefactors in Eq. (26), the ratio of the boundary layer and the bulk dissipation remains above unity, implying that the boundary layer dissipation is larger than the bulk dissipation, although they are of the same order. As shown in Figs. 4(c) and 4(d), the boundary layer dissipation is approximately 3-4 times greater than the bulk dissipation. This is unlike viscous dissipation for $\mathrm{Pr}$ $=1$, where the dissipation in the bulk is greater, albeit marginally, than that in the boundary layers. ${ }^{34}$ This is because while the temperature is fairly constant in the bulk (except for a few regions of localized plumes), the velocity in the bulk is not so, as illustrated in 

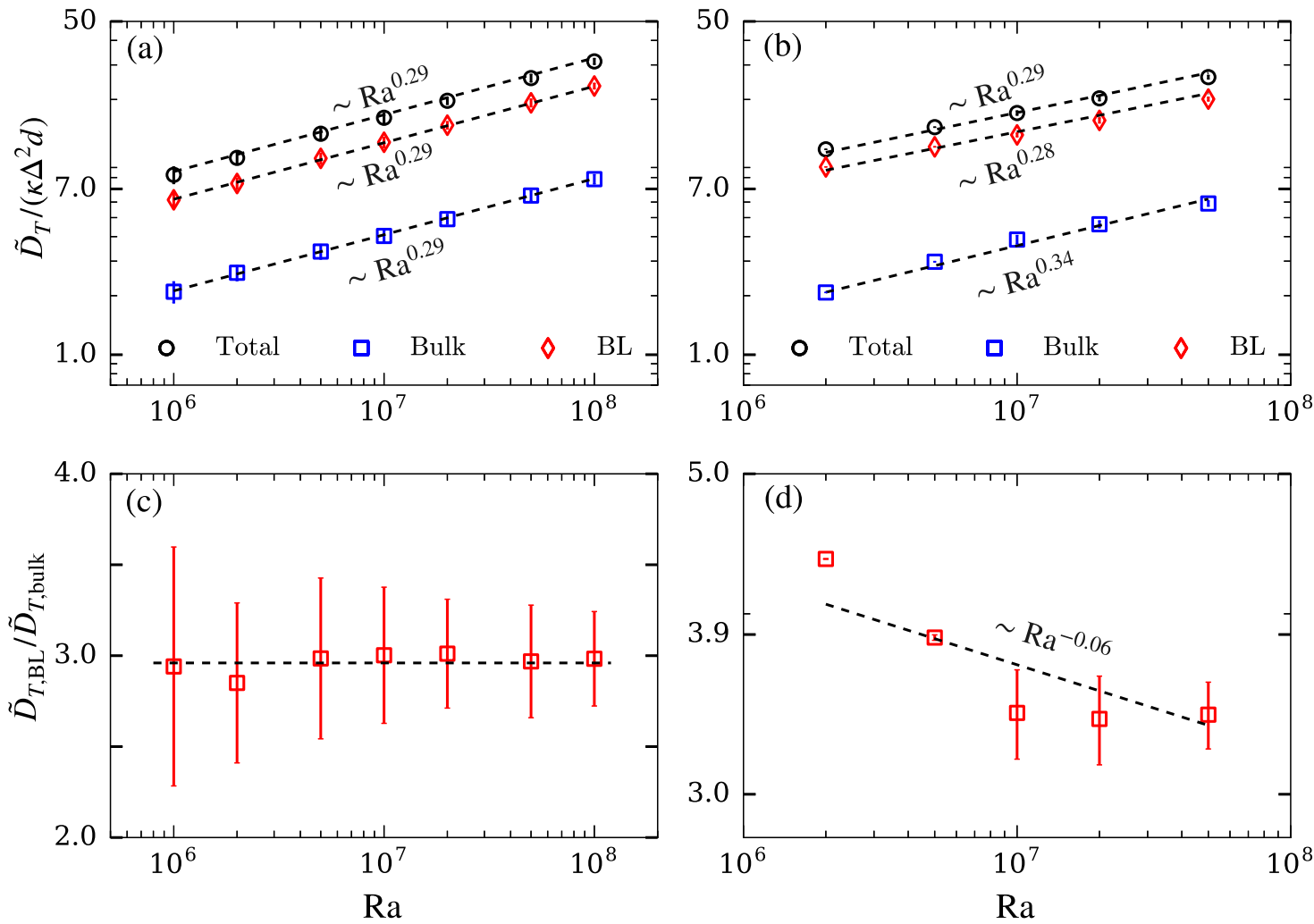

FIG. 4. For (a) $\operatorname{Pr}=1$ and (b) $\operatorname{Pr}=100$ : plots of thermal dissipation rates $\tilde{D}_{T}$-total, bulk, and in the boundary layers (BL)-vs $\operatorname{Ra}$. For $(\mathrm{c}) \operatorname{Pr}=1$ and (d) $\operatorname{Pr}=100$ : plots of the dissipation rate ratio, $\tilde{D}_{T, \mathrm{BL}} / \tilde{D}_{T, \text { bulk }}$, vs Ra. The error bars represent the standard deviation of the dataset with respect to the temporal average.

Fig. 5. Here, we show the temperature density plot superimposed with velocity vector plot on the $x-z$ plane at $y=d / 2$, for $\mathrm{Ra}=10^{8}$, $\operatorname{Pr}=1$. Clearly, the velocity fluctuations are large near the walls (just outside the viscous boundary layers) but small near the center. On the other hand, $T \approx 0.5$ in the bulk. Thus, the velocity gradients in

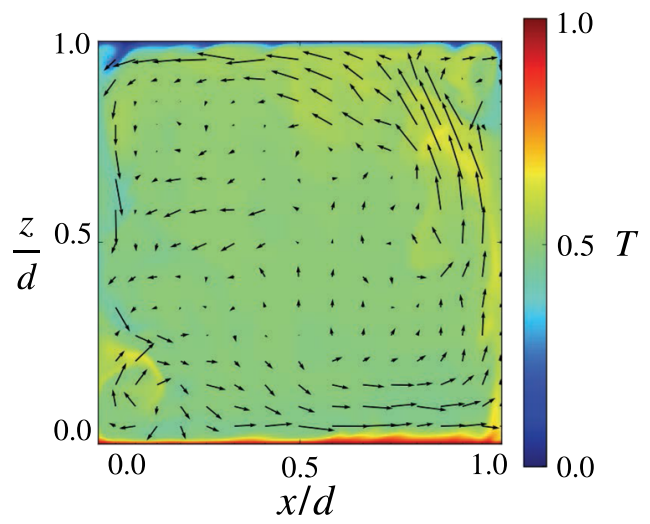

FIG. 5. For $\mathrm{Ra}=10^{8}, \mathrm{Pr}=1$ : Density plot of temperature field, superimposed with velocity vector plot, on the $x-z$ plane at $y=d / 2$. The temperature is approximately constant in the bulk, unlike the velocity fluctuations. the bulk are more pronounced than the temperature gradients; this results in stronger viscous dissipation compared to thermal dissipation in the bulk. However, one must note that for $\operatorname{Pr}=100$, the viscous boundary layers will occupy almost the entire volume; thus, the viscous dissipation in the boundary layers will be dominant. Also, we need to carefully simulate low Pr convection to find out whether bulk or boundary layer dissipation dominates in this regime.

The dominance of the total thermal dissipation in the boundary layers has been reported previously for convection in a slender cylindrical cell ${ }^{24}$ and for two-dimensional convection.

\section{Spatial intermittency of thermal dissipation rate}

In this subsection, we will study the intermittency of the local thermal dissipation rate $\epsilon_{T}(\mathbf{r})$. Since $\delta_{T} / d \ll 1$ (see Fig. 1), the boundary layers occupy a much smaller volume than the bulk. Therefore, $\epsilon_{T}(\mathbf{r})$ is much stronger in the boundary layers than in the bulk.

We compute the probability distribution functions (PDFs) of $\epsilon_{T}^{*}(\mathbf{r})=\epsilon_{T}(\mathbf{r}) / \epsilon_{T}$ in the entire volume, bulk and boundary layers to quantify the spatial intermittency of thermal dissipation rate. The PDFs are computed for $\mathrm{Ra}=5 \times 10^{7}$ for both $\mathrm{Pr}=1$ and 100. We plot these quantities in Fig. 6(a) for $\operatorname{Pr}=1$ and in Fig. 6(b) for $\operatorname{Pr}=100$. From the inset of Fig. 6(a), we observe that for $\operatorname{Pr}=1, P\left(\epsilon_{T, \text { bulk }}^{*}\right)$ $\gg P\left(\epsilon_{T, \mathrm{BL}}^{*}\right)$ for $\epsilon_{T}^{*}<10$, while $P\left(\epsilon_{T, \text { bulk }}^{*}\right) \ll P\left(\epsilon_{T, \mathrm{BL}}^{*}\right)$ for $\epsilon_{T}^{*}>10$. 

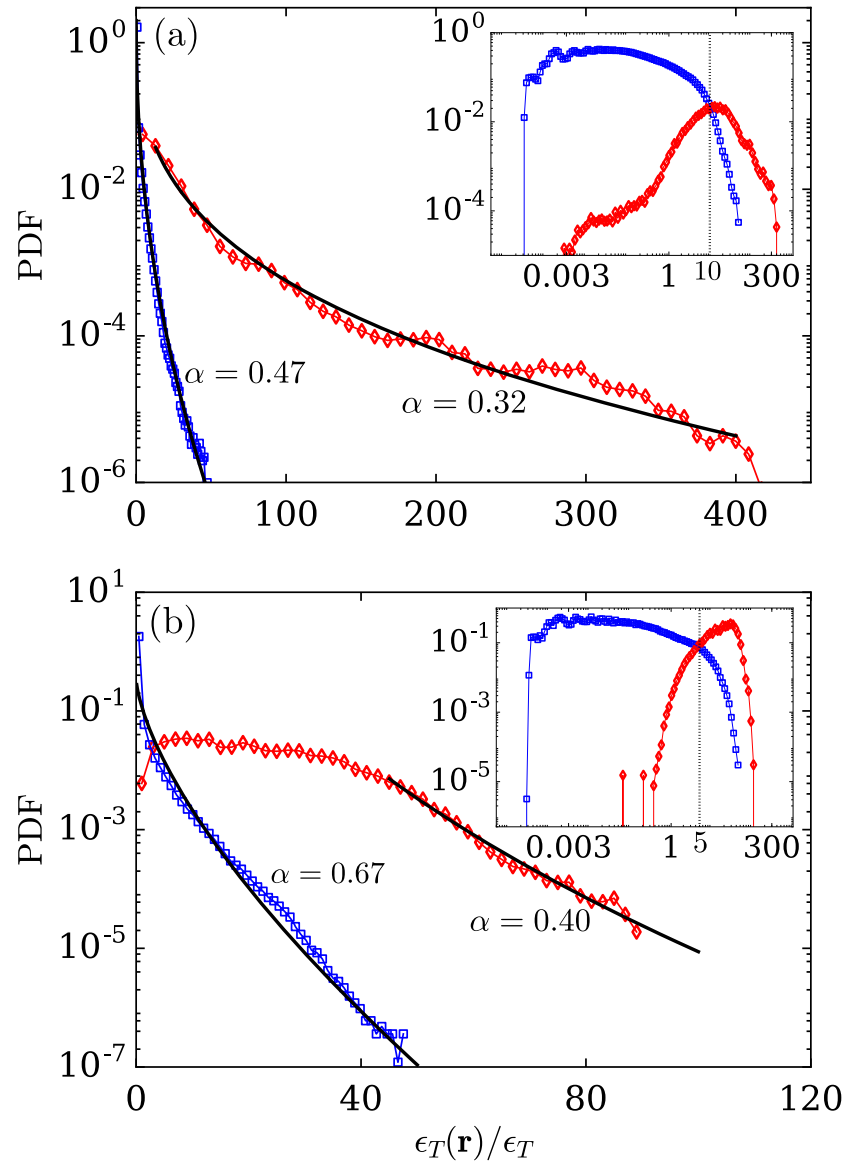

FIG. 6. For $\mathrm{Ra}=5 \times 10^{7}$ and (a) $\mathrm{Pr}=1$ and (b) $\mathrm{Pr}=100$ : probability distribution functions (PDF) of normalized local dissipation rate $\epsilon_{T}(\mathbf{r}) / \epsilon_{T}$ in the bulk (blue squares) and in the boundary layers (red diamonds). The PDFs of both $\epsilon_{T, B L}$ and $\epsilon_{T, \text { bulk }}$ fit well with stretched exponential curves (black solid lines). The insets in (a) and (b) show the log-log plots of the PDFs of $\epsilon_{T}(\mathbf{r})$ in the bulk and the boundary layers.

This clearly shows that thermal dissipation is weak in the bulk and strong in the boundary layers. We observe a similar behavior for $\mathrm{Pr}$ $=100$, but with cutoff $\epsilon_{T}^{*} \approx 5$ [see the inset of Fig. 6(b)].

It has been analytically shown by Chertkov, Falkovich, and Kolokolov ${ }^{46}$ that the passive scalar dissipation has a stretched exponential distribution. This profile is given by $P\left(\epsilon_{T}\right) \sim \beta \exp \left(-m \epsilon_{T}^{* \alpha}\right)$ for $\epsilon_{T}^{*} \gg 1$. Interestingly, the PDFs of thermal dissipation for RBC are also stretched exponential for both bulk and boundary layers. Our observation is consistent with earlier studies. ${ }^{35,44,47}$ For bulk dissipation, the stretching exponent $\alpha=0.47$ for $\operatorname{Pr}=1$ and $\alpha=0.67$ for $\operatorname{Pr}=100$. The corresponding exponents for the boundary layers are 0.32 and 0.40 , respectively.

Clearly, for both Pr, the tails of the PDFs are stretched more for the boundary layer dissipation. This is expected because extreme events are more frequent in the boundary layers than in the bulk; note that $\epsilon_{T}(\mathbf{r})$ is stronger in the boundary layers. Furthermore, for both bulk and boundary layer dissipation, $\alpha$ 's are smaller for $\operatorname{Pr}=1$. Thus, the tails of the PDFs are stretched more for $\operatorname{Pr}=1$, implying stronger spatial intermittency of thermal dissipation for the lower $\mathrm{Pr}$ fluid. This is because for $\operatorname{Pr}=1$, convection is more turbulent than that for $\operatorname{Pr}=100$, causing the temperature fluctuations to be more pronounced for the former.

\section{CONCLUSIONS}

In this paper, we present scaling relations for the thermal dissipation rate in the bulk and in the boundary layers in turbulent convection. Using numerical simulations of RBC, we show that compared to passive scalar turbulence, the thermal dissipation rate in the bulk is suppressed by a factor of $\mathrm{Ra}^{-0.22}$ for $\mathrm{Pr}=1$ and $\mathrm{Ra}^{-0.25}$ for $\operatorname{Pr}=100$. Furthermore, unlike viscous dissipation, the total thermal dissipation in the boundary layers is greater than that in the bulk. The ratio of the boundary layer and the bulk dissipation is roughly constant for $\operatorname{Pr}=1$ and decreases weakly with $\mathrm{Ra}$ for $\operatorname{Pr}=100$.

We also show that the probability distribution functions of thermal dissipation rate, both in the bulk and in the boundary layers, are stretched exponential, similar to passive scalar dissipation. The stretching exponent for the PDFs of boundary layer dissipation is lower than that of bulk dissipation, implying that extreme events occur more often in the boundary layers than in the bulk. We also show that the spatial intermittency of thermal dissipation is stronger for lower Pr fluids.

The results presented in this paper are important for modeling thermal convection. For example, we may need to incorporate the suppression of thermal dissipation in the bulk in the scaling analysis for $\mathrm{Pe}$ and $\mathrm{Nu}$. Thus far, our analysis has been for $\operatorname{Pr} \geq 1$. We need to extend them to low Pr convection for a comprehensive modeling of thermal convection.

\section{ACKNOWLEDGMENTS}

We thank A. Pandey, A. Guha, and R. Samuel for useful discussions. Our numerical simulations were performed on Shaheen II at KAUST Supercomputing Laboratory, Saudi Arabia, under the Project No. k1052. This work was supported by the research Grant No. PLANEX/PHY/2015239 from Indian Space Research Organisation, India, and by the Department of Science and Technology, India (Grant No. INT/RUS/RSF/P-03), and the Russian Science Foundation, Russia (Grant No. RSF-16-41-02012), for the Indo-Russian project.

\section{REFERENCES}

${ }^{1}$ A. M. Obukhov, "Structure of the temperature field in a turbulent flow," Isv. Geogr. Geophys. Ser. 13, 58-69 (1949).

${ }^{2}$ S. Corrsin, "On the spectrum of isotropic temperature fluctuations in an isotropic turbulence,” J. Appl. Phys. 22, 469-473 (1951).

${ }^{3}$ M. Lesieur, Turbulence in Fluids (Springer-Verlag, Dordrecht, 2008).

${ }^{4}$ M. K. Verma, Physics of Buoyant Flows (World Scientific, Singapore, 2018).

${ }^{5}$ G. Ahlers, S. Grossmann, and D. Lohse, "Heat transfer and large scale dynamics in turbulent Rayleigh-Bénard convection," Rev. Mod. Phys. 81, 503-537 (2009).

${ }^{6}$ D. Lohse and K.-Q. Xia, "Small-scale properties of turbulent Rayleigh-Bénard convection,” Annu. Rev. Fluid Mech. 42, 335-364 (2010).

${ }^{7}$ M. K. Verma, A. Kumar, and A. Pandey, "Phenomenology of buoyancy-driven turbulence: Recent results,” New J. Phys. 19, 025012 (2017).

${ }^{8}$ B. I. Shraiman and E. D. Siggia, "Heat transport in high-Rayleigh-number convection," Phys. Rev. A 42, 3650-3653 (1990). 
${ }^{9}$ R. H. Kraichnan, "Turbulent thermal convection at arbitrary Prandtl number," Phys. Fluids 5, 1374-1389 (1962).

${ }^{10}$ M. K. Verma, P. K. Mishra, A. Pandey, and S. Paul, "Scalings of field correlations and heat transport in turbulent convection," Phys. Rev. E 85, 016310 (2012).

${ }^{11}$ S. Grossmann and D. Lohse, "Scaling in thermal convection: A unifying theory," J. Fluid Mech. 407, 27-56 (2000).

${ }^{12}$ S. Grossmann and D. Lohse, "Thermal convection for large Prandtl numbers," Phys. Rev. Lett. 86, 3316-3319 (2001).

${ }^{13}$ W. V. R. Malkus, "The heat transport and spectrum of thermal turbulence," Proc. R. Soc. London, Ser. A 225, 196-212 (1954).

${ }^{14}$ B. Castaing, G. Gunaratne, L. P. Kadanoff, A. Libchaber, and F. Heslot, "Scaling of hard thermal turbulence in Rayleigh-Bénard convection," J. Fluid Mech. 204, $1-30$ (1989).

${ }^{15}$ S. Grossmann and D. Lohse, "Prandtl and Rayleigh number dependence of the Reynolds number in turbulent thermal convection," Phys. Rev. E 66, 016305 (2002).

${ }^{16} \mathrm{X}$.-L. Qiu and P. Tong, "Temperature oscillations in turbulent Rayleigh-Bénard convection," Phys. Rev. E 66, 026308 (2002).

${ }^{17}$ X.-L. Qiu, X.-D. Shang, P. Tong, and K.-Q. Xia, "Velocity oscillations in turbulent Rayleigh-Bénard convection," Phys. Fluids 16, 412-423 (2004).

${ }^{18}$ E. Brown, D. Funfschilling, and G. Ahlers, "Anomalous Reynolds-number scaling in turbulent Rayleigh-Bénard convection," J. Stat. Mech.: Theory Exp. 2007, P10005.

${ }^{19}$ D. Funfschilling, E. Brown, A. Nikolaenko, and G. Ahlers, "Heat transport by turbulent Rayleigh-Bénard convection in cylindrical samples with aspect ratio one and larger," J. Fluid Mech. 536, 145-154 (2005).

${ }^{20}$ A. Nikolaenko, E. Brown, D. Funfschilling, and G. Ahlers, "Heat transport by turbulent Rayleigh-Bénard convection in cylindrical cells with aspect ratio one and less," J. Fluid Mech. 523, 251-260 (2005).

${ }^{21}$ X. He, D. Funfschilling, E. Bodenschadtz, and G. Ahlers, "Heat transport by turbulent Rayleigh-Bénard convection for $\operatorname{Pr} \simeq 0.8$ and $4 \times 10^{11} \lesssim \mathrm{Ra} \lesssim 2 \times 10^{14}$ : Ultimate-state transition for aspect ratio $\Gamma=1.00$," New J. Phys. 14, 063030 (2012).

${ }^{22}$ G. Ahlers, X. He, D. Funfschilling, and E. Bodenschadtz, "Heat transport by turbulent Rayleigh-Bénard convection for $\operatorname{Pr} \simeq 0.8$ and $3 \times 10^{12} \lesssim \mathrm{Ra} \lesssim 10^{15}$ : Aspect ratio $\Gamma=0.50$," New J. Phys. 14, 103012 (2012).

${ }^{23} \mathrm{M}$. Vial and R. H. Hernándes, "Feedback control and heat transfer measurements in a Rayleigh-Bénard convection cell," Phys. Fluids 29, 074103 (2017).

${ }^{24} \mathrm{R}$. Verzicco and R. Camussi, "Numerical experiments on strongly turbulent thermal convection in a slender cylindrical cell," J. Fluid Mech. 477, 19-49 (2003). ${ }^{25}$ J. D. Scheel, E. Kim, and K. R. White, "Thermal and viscous boundary layers in turbulent Rayleigh-Bénard convection," J. Fluid Mech. 711, 281-305 (2012).

${ }^{26}$ J. D. Scheel and J. Schumacher, "Local boundary layer scales in turbulent Rayleigh-Bénard convection," J. Fluid Mech. 758, 344-373 (2014).

${ }^{27} \mathrm{~F}$. Waleffe, A. Boonkasame, and L. M. Smith, "Heat transport by coherent Rayleigh-Bénard convection," Phys. Fluids 27, 051702 (2015).
${ }^{28}$ M. K. Verma, S. C. Ambhire, and A. Pandey, "Flow reversals in turbulent convection with free-slip walls," Phys. Fluids 27, 047102 (2015).

${ }^{29}$ W.-F. Zhou and J. Chen, "Letter: Similarity model for corner roll in turbulent Rayleigh-Bénard convection,” Phys. Fluids 30, 111705 (2018).

${ }^{30}$ A. Pandey and M. K. Verma, "Scaling of large-scale quantities in RayleighBénard convection," Phys. Fluids 28, 095105 (2016).

${ }^{31}$ A. Pandey, A. Kumar, A. G. Chatterjee, and M. K. Verma, "Dynamics of largescale quantities in Rayleigh-Bénard convection," Phys. Rev. E 94, 053106 (2016).

${ }^{32}$ B. A. Puthenveettil and J. H. Arakeri, "Plume structure in high-Rayleighnumber convection," J. Fluid Mech. 542, 217-249 (2005).

${ }^{33}$ B. A. Puthenveettil, G. Ananthakrishna, and J. H. Arakeri, "The multifractal nature of plume structure in high-Rayleigh-number convection," J. Fluid Mech. 526, 245-256 (2005).

${ }^{34}$ S. Bhattacharya, A. Pandey, A. Kumar, and M. K. Verma, "Complexity of viscous dissipation in turbulent thermal convection," Phys. Fluids 30, 031702 (2018).

${ }^{35}$ Y. Zhang, Q. Zhou, and C. Sun, "Statistics of kinetic and thermal energy dissipation rates in two-dimensional turbulent Rayleigh-Bénard convection," J. Fluid Mech. 814, 165-184 (2017).

${ }^{36} \mathrm{~S}$. Chandrasekhar, Hydrodynamic and Hydromagnetic Stability (Oxford University Press, Oxford, 2013).

${ }^{37}$ A. Kumar and M. K. Verma, "Applicability of Taylor's hypothesis in thermally driven turbulence," R. Soc. Open Sci. 5, 172152 (2018).

${ }^{38} \mathrm{H}$. Jasak, A. Jemcov, Z. Tukovic et al., "OpenFOAM: A C++ library for complex physics simulations," in International Workshop on Coupled Methods in Numerical Dynamics (IUC Dubrovnik, Croatia, 2007), Vol. 1000, pp. 1-20.

${ }^{39} \mathrm{G}$. Grötzbach, "Spatial resolution requirements for direct numerical simulation of the Rayleigh-Bénard convection," J. Comput. Phys. 49, 241-264 (1983).

${ }^{40}$ N. Shi, M. S. Emran, and J. Schumacher, "Boundary layer structure in turbulent Rayleigh-Bénard convection,” J. Fluid Mech. 706, 5-33 (2012).

${ }^{41} \mathrm{G}$. K. Batchelor, "Small-scale variation of convected quantities like temperature in turbulent fluid Part 1. General discussion and the case of small conductivity," J. Fluid Mech. 5, 113-133 (1959).

${ }^{42} \mathrm{~S}$. Wagner and O. Shishkina, "Aspect-ratio dependency of Rayleigh-Benard convection in box-shaped containers," Phys. Fluids 25, 085110 (2013).

${ }^{43}$ A. Pandey, M. K. Verma, and P. K. Mishra, "Scaling of heat flux and energy spectrum for very large Prandtl number convection," Phys. Rev. E 89, 023006 (2014).

${ }^{44}$ M. S. Emran and J. Schumacher, "Fine-scale statistics of temperature and its derivatives in convective turbulence," J. Fluid Mech. 611, 13-34 (2008).

${ }^{45}$ O. Shishkina, M. S. Emran, S. Grossmann, and D. Lohse, "Scaling relations in large-Prandtl-number natural thermal convection," Phys. Rev. Fluids 2, 103502 (2017).

${ }^{46} \mathrm{M}$. Chertkov, G. Falkovich, and I. Kolokolov, "Intermittent dissipation of a passive scalar in turbulence," Phys. Rev. Lett. 80, 2121-2124 (1998).

${ }^{47} \mathrm{X}$. He and P. Tong, "Measurements of the thermal dissipation field in turbulent Rayleigh-Bénard convection," Phys. Rev. E 79, 026306 (2009). 